

\title{
BLOQUES DE TIERRA COMPRIMIDA (BTC) ESTABILIZADOS CON CAL Y CEMENTO. EVALUACIÓN DE SU IMPACTO AMBIENTAL Y SU RESISTENCIA A COMPRESIÓN
}

\author{
COMPRESSED EARTH BLOCKS (CEB) STABILIZED WITH LIME AND \\ CEMENT. EVALUATION OF BOTH THEIR ENVIRONMENTAL IMPACT \\ AND COMPRESSIVE STRENGTH
}

\author{
SANTIAGO PEDRO CABRERA \\ Ingeniero Civil \\ Becario Doctoral de la Facultad Regional Santa Fe \\ Universidad Tecnológica Nacional \\ Santa Fe, Argentina \\ https://orcid.org/0000-0003-1300-4927 \\ spcabrera@outlook.com \\ EDGARDO JONATHAN SUÁREZ DOMÍNGUEZ \\ Doctor en Química \\ Investigador de la Facultad de Arquitectura, Diseño y Urbanismo \\ Universidad Autónoma de Tamaulipas \\ Ciudad Victoria, México \\ https://orcid.org/0000-0002-1342-5732 \\ edgardo.suarez@docentes.uat.edu.mx
}

\author{
YOLANDA GUADALUPE ARANDA JIMÉNEZ \\ Doctora en Arquitectura \\ Investigadora de la Facultad de Arquitectura, Diseño y Urbanismo \\ Universidad Autónoma de Tamaulipas \\ Ciudad Victoria, México \\ https://orcid.org/0000-0002-5026-507X \\ yoli212@yahoo.com.mx \\ RODOLFO ROTONDARO \\ Doctor en Arquitectura \\ Investigador de la Facultad de Arquitectura, \\ Diseño y Urbanismo / CONICET \\ Universidad de Buenos Aires, Buenos Aires, Argentina \\ https://orcid.org/0000-0001-9861-8340 \\ rodolforotondaro@gmail.com
}

\section{RESUMEN}

En este trabajo se presenta la evaluación del impacto ambiental y la resistencia a compresión de Bloques de Tierra Comprimida (BTC) estabilizados con cal aérea hidratada y cemento Portland. Para esa labor, se fabricaron 12 series de bloques estabilizados con diferentes proporciones de cal y cemento y se empleó la metodología del Análisis de Ciclo de Vida (ACV). Tras la realización de los ensayos y las simulaciones pudo concluirse que, usando suelos y arena característicos de la ciudad de Santa Fe (Argentina), estabilizados con determinados porcentajes de cemento Portland -comprendidos entre el 5 y el 10\% en peso- pueden producirse BTC con niveles de resistencia suficientes para ser utilizados en muros de carga y, de esa forma, minimizar el impacto ambiental negativo asociado a su fabricación. Se concluye, además, que la estabilización con cal aérea no incrementa la resistencia a compresión de los BTC y aumenta, por el contrario, de manera significativa el impacto negativo de éstos sobre el medio ambiente.

\section{Palabras clave}

tierra, estabilización, resistencia de materiales, impacto ambiental

\section{ABSTRACT}

This work presents the evaluation of the environmental impact and compressive strength of Compressed Earth Blocks (CEB) stabilized with hydrated aerial lime and Portland cement. For this, 12 series of blocks stabilized with different proportions of lime and cement were manufactured and the Life Cycle Analysis (LCA) methodology was used. After conducting these assays and simulations, it could be concluded that, using earth and sand typical of the city of Santa Fe (Argentina), stabilized with certain percentages of Portland cement between 5 and $10 \%$ in weight, CEB can be produced with sufficient

levels of strength for them to be used in load-bearing walls, in this way minimizing the negative environmental impact associated with their manufacturing. It is also concluded that the stabilization with aerial lime does not increase the CEB's compressive strength and, on the contrary, significantly increases their negative impact on the environment. 


\section{INTRODUCCIÓN}

El Bloque de Tierra comprimida o BTC es un mampuesto fabricado mediante la compresión o el prensado de suelo estabilizado que se encuentra contenido en el interior de una prensa mecánica o hidráulica (Neves y Borges Farías, 2011). Estas prensas pueden ser manuales, par bajas demandas de producción, o bien, automáticas, para sistemas industrializados (González y Cabrera, 2017). La tecnología del BTC inició su desarrollo en Colombia a comienzos de la década de los 50, de la mano del Centro Interamericano de Vivienda y Planeamiento -CINVA-, como una alternativa económica de elementos constructivos que, actualmente, se considera una de las tecnologías latinoamericanas más difundidas en el mundo (Angulo y Carreño, 2017).

La mampostería con BTC es, en efecto, una técnica de construcción económica que presenta mejores propiedades de resistencia y durabilidad que las construidas con adobe, y una gran potencialidad de industrialización de sus unidades (Herrera Villa, 2018). Además, estos bloques poseen varias ventajas que les permiten responder a los problemas energéticos y climáticos actuales, por ser elementos fabricados con materiales de baja energía incorporada (Bradley, Gohnert y Bulovic, 2018), en comparación con los ladrillos de arcilla cocida y arena-cemento, de modo que reducen el total de energía requerida para la construcción y el transporte, lo cual se debe, en gran medida, a que la tierra es un recurso natural abundante y reciclable (Ben Mansour, Ogam, Jelidi, Cherif y Ben Jabrallah, 2017; Hegyi, Dico y Catalan, 2016).

Al igual que las demás técnicas de construcción con tierra, los BTC presentan dos limitaciones principales:

- Si las características de la tierra empleada para su fabricación no son las adecuadas, su resistencia a compresión no será suficiente para cumplir funciones estructurales (Ouedraogo, Aubert, Tribout y Escadeilas, 2020);

- Independientemente del tipo de tierra empleada para su fabricación, exhiben algunos problemas de durabilidad: se degradan frente a ciertos fenómenos atmosféricos, en especial el agua (Laborel-Préneron, Aubert, Jean-Emmanuel Magniont, Maillard y Poirier, 2016).

Ambas limitaciones pueden minimizarse e, incluso, eliminarse empleando pequeños porcentajes de estabilizantes químicos durante su fabricación, los cuales mejoran sus propiedades físico-mecánicas, aumentando su resistencia y durabilidad (Guzmán y Iñiguez, 2016).

Diversos aditivos han sido empleados en la estabilización de los BTC: desde sustancias naturales como acíbar de sábila (Aranda Jiménez y Suárez-Domínguez, 2014), caseína y celulosa (Vissac, Bourges y Gandreau, 2017), hasta derivados del petróleo como bitúmenes naturales y emulsiones asfálticas (Van Damme y Houben, 2018). Sin embargo, desde los orígenes de esta tecnología, el estabilizante más empleado ha sido el cemento Portland (Elahi, Shahriar, Alam y Abedin, 2020), tal es el caso que en numerosas regiones de Argentina y Latinoamérica suelen ser conocidos como "Bloques de Suelo Cemento", léxico muy influenciado por la ingeniera de caminos.

A pesar de las buenas prestaciones que poseen los BTC estabilizados con cemento, debe considerarse que, junto con el elevado costo económico de este aditivo, su fabricación requiere también de elevados procesos de transformación térmica -que alcanzan los $1.450^{\circ} \mathrm{C}$ - y libera enormes cantidades de $\mathrm{CO}_{2}$ a la atmósfera. Se estima que por cada tonelada de cemento Portland producida son liberadas a la atmósfera 0.86 toneladas de $\mathrm{CO}_{2}$ (Guilarducci, 2018).

Otro aditivo frecuentemente empleado en la estabilización de BTC es la cal, tanto aérea como hidráulica (Malkanthi, Balthazaar y Perera, 2020), cuyo impacto ambiental es significativamente menor al del cemento Portland por los siguientes motivos:

- La temperatura requerida para su fabricación es de aproximadamente $900^{\circ} \mathrm{C}$ (Maddalena, Roberts y Hamilton, 2018);

- Puede ser producida a pequeña escala y de manera artesanal (Guapi Cepeda y Yagual Flores, 2017);

- La cal aérea tiene la propiedad de absorber durante su proceso de endurecimiento (carbonatación) gran parte del CO2 liberado a la atmósfera durante su proceso de fabricación (Qiu, 2020).

Numerosos países cuentan con normas técnicas específicas para la construcción con tierra, no obstante, son pocos los que poseen normas de ensayo específicas para BTC publicadas por Organismos Oficiales de Normalización, entre los que destacan Brasil, Colombia, Francia, España y México (Cabrera, González y Rotondaro, 2020).

En este contexto, el objetivo general de la investigación expuesta consistió en evaluar las propiedades mecánicas y ambientales de los BTC estabilizados con cal aérea hidratada y contrastarlas con las de sus homólogos estabilizados con cemento Portland. Para ello, se proponen los siguientes objetivos particulares:

- Determinar la resistencia media a compresión de BTC estabilizados con diferentes porcentajes de cal aérea hidratada y cemento Portland;

- Correlacionar la resistencia media a compresión de los BTC con el porcentaje y tipo de estabilizante empleado.

- Calcular el impacto ambiental de los BTC estabilizados con diferentes proporciones de cal y cemento. 


\begin{tabular}{|c|c|c|c|c|c|c|c|}
\hline \multicolumn{2}{|c|}{ Tamiz } & $\mathbf{4 . 7 5} \mathbf{~ m m}$ & $\mathbf{0 . 4 2 5} \mathbf{~ m m}$ & $\mathbf{0 . 2 5 0} \mathbf{~ m m}$ & $\mathbf{0 . 1 5 0} \mathbf{~ m m}$ & $\mathbf{0 . 0 7 5} \mathbf{~ m m}$ & $\mathbf{0 . 0 0 2} \mathbf{~ m m}$ \\
\hline \multirow{2}{*}{ Pasante (\%) } & Tierra & 0.0 & 86.0 & - & - & 57.0 & 29.0 \\
\cline { 2 - 8 } & Arena & 100 & 96.2 & 89.3 & 48.7 & 0.6 & 0.0 \\
\hline
\end{tabular}

Tabla 1. Granulometría de la materia prima. Fuente: Elaboración de los autores.

\section{METODOLOGÍA}

\section{FABRICACIÓN DE LOS BTC}

La tierra empleada en la fabricación de los BTC provino de una cantera emplazada en la comuna de Monte Vera (Santa Fe, Argentina) clasificada como un limo arcilloso de baja plasticidad "CL ML" con $29 \%$ de arcilla (caolinita e ilita) y un índice de contracción lineal del $4 \%$. Con intenciones de mejorar la curva granulométrica de la tierra e incrementar así la resistencia a compresión de los BTC producidos (Sitton, Zeinali, Heidarian y Story, 2018), fue mezclada con arena proveniente del río Paraná y constituida principalmente por cuarzo $\left(\mathrm{SiO}_{2}\right)$. La distribución granulométrica de la tierra y la arena empleadas en la fabricación de los BTC puede apreciarse en la Tabla 1.

Para determinar la resistencia a compresión de los BTC estabilizados con diferentes proporciones de cal y cemento se fabricaron en laboratorio 12 series de 5 BTC cada una, manteniendo fija la relación tierra/ arena. Se produjeron bloques de $25.0 \times 12.5 \times 6.25 \mathrm{~cm}$ con dos perforaciones verticales de $6.0 \mathrm{~cm}$ de diámetro cada una, empleando para ello una prensa hidráulica Eco Brava desarrollada por la compañía brasileña "Eco Máquinas". Todas las series fueron confeccionadas con una humedad del 12.5\%, considerando el peso seco de los materiales. Los estabilizantes usados fueron cal aérea hidratada marca "Andina" y cemento Portland compuesto CPC 40 marca "Holcim". Finalmente, en la Tabla 2 se expresan las diferentes dosificaciones utilizadas.

Los BTC producidos fueron cortados en 2 mitades, generando así 2 probetas de $12.5 \times 12.5 \times 6.5 \mathrm{~cm}$ por BTC, de las que luego se seleccionaron, de manera aleatoria, 6 por serie, para someterlas al ensayo de resistencia a compresión estipulado por la norma mexicana NMX-C-508 (ONNCCE, 2015): ensayo sin encabezado y velocidad de carga variable (para que el ensayo dure entre 1 y 2 minutos). Todas las probetas se ensayaron secas con 28 días de edad y curadas por 7 días (con excepción de las series sin estabilizante) a una humedad relativa del $100 \%$, permaneciendo luego

\begin{tabular}{|c|c|c|c|c|c|}
\hline \multicolumn{2}{|c|}{ Serie } & $\begin{array}{c}\text { Tierra } \\
(\%)\end{array}$ & $\begin{array}{c}\text { Arena } \\
(\%)\end{array}$ & $\begin{array}{l}\text { Cal } \\
(\%)\end{array}$ & $\begin{array}{c}\text { Cemento } \\
(\%)\end{array}$ \\
\hline \multirow{6}{*}{ 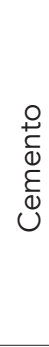 } & Cto. $0 \%$ & 50,0 & 50,0 & - & 0,0 \\
\hline & Cto. $2,5 \%$ & 48,75 & 48,75 & - & 2,5 \\
\hline & Cto. 5\% & 47,5 & 47,5 & - & 5,0 \\
\hline & Cto. $10 \%$ & 45,0 & 45,0 & - & 10,0 \\
\hline & Cto. 15\% & 42,5 & 42,5 & - & 15,0 \\
\hline & Cto. $20 \%$ & 40,0 & 40,0 & - & 20,0 \\
\hline \multirow{6}{*}{$\bar{U}$} & Cal 0\% & 70,0 & 30,0 & 0,0 & - \\
\hline & Cal 2,5\% & 68,25 & 29,25 & 2,5 & - \\
\hline & Cal 5\% & 66,5 & 28,5 & 5,0 & - \\
\hline & Cal 10\% & 63,0 & 27,0 & 10,0 & - \\
\hline & Cal 15\% & 59,5 & 25,5 & 15,0 & - \\
\hline & Cal 20\% & 56,0 & 24,0 & 20,0 & - \\
\hline
\end{tabular}

Tabla 2. Dosificación en peso de las diferentes series de BTC. Fuente: Elaboración de los autores.

21 días en ambiente de laboratorio, a una humedad relativa del $55 \%$ y $24^{\circ} \mathrm{C}$ de temperatura.

Con el fin de evaluar los resultados obtenidos y determinar si las resistencias medias a compresión de cada serie eran estadísticamente diferentes entre sí, se realizó un análisis de varianzas ANOVA y un "análisis de parejas de Tukey", empleando el software estadístico MiniTab'.

\section{ANÁLISIS DE CICLO DE VIDA}

Para determinar el impacto ambiental de los BTC estabilizados con diferentes contenidos de cal y cemento, se empleó la metodología de Análisis de Ciclo de Vida (ACV) propuesta por la norma IRAM-ISO 14040 (IRAM, 2017), la cual permite caracterizar y cuantificar los diversos impactos ambientales potenciales asociados a cada una de las etapas de la vida útil de un producto o sistema (Carretero-Ayuso y García-Sanz-Calcedo, 2018). En la realización del análisis de inventario propuesto por la IRAM-ISO 14040, se utilizó el software SimaPro9 (Copyright Pré, 2019). 


\section{UNIDAD DE ANÁLISIS}

En este marco, se adoptaron como unidades funcionales BTC individuales de $15 \times 30 \times 7.5 \mathrm{~cm}$ con dos agujeros de $6 \mathrm{~cm}$ de diámetro (geometría de los BTC producidos por la empresa Mobak en Santa Fe, Argentina) y una masa de $4 \mathrm{~kg}$, cada uno de ellos con un contenido de cal o cemento diferente, coincidentes con la dosificación de los bloques ensayados a compresión (ver Tabla 2).

\section{LÍMITES DEL SISTEMA}

El alcance del estudio se limitó al enfoque denominado "de la cuna a la puerta", mediante el cual se consideran únicamente los flujos de entrada y salida de las etapas comprendidas entre la extracción de las materias primas necesarias para la fabricación de los bloques hasta que éstos se encuentran terminados y listos para insertarse en el mercado, fuera de la planta de producción (Curadelli, López, Piastrellini, Arena y Civit, 2019). Para cuantificar el impacto asociado al transporte de las materias primas desde su punto de extracción o adquisición se consideró lo siguiente:

- Unidad productiva: El modelo de análisis se basó en la Fábrica de BTC Mobak, ubicada en la comuna de Arroyo Leyes (Santa Fe), cuya capacidad productiva es de 3.000 BTC diarios.

- Tierra: La cantera de extracción de la tierra se encuentra en la comuna de Monte Vera (Santa Fe), a $35 \mathrm{~km}$ de distancia de la fábrica.

- Arena: La adquisición de la arena empleada en la fabricación de los bloques se llevó a cabo en una arenera de la Ciudad de Santa Fe, a $19 \mathrm{~km}$ de distancia de la unidad productiva.

- Estabilizantes: Se consideró la utilización de cal aérea hidratada en bolsas de $25 \mathrm{~kg}$ y cemento Portland en bolsas de $50 \mathrm{~kg}$, ambos adquiridos en un corralón localizado al norte de la ciudad de Santa $\mathrm{Fe}$, a $35 \mathrm{~km}$ de la fábrica.

- Transporte: Para el transporte de la tierra y la arena se consideró la utilización de un camión tipo euro3 de 16-32 tn de capacidad; mientras que, para el transporte de los estabilizantes, un camión euro3 de 7.5-16 tn de capacidad. Estas categorías de vehículos se adoptaron siguiendo los requisitos estipulados por la Norma 5 de la Unión Europea (UE, 2007).
- Extracción y producción de materia prima: con el objetivo de cuantificar el impacto asociado a la extracción y comercialización de la tierra y la arena, y la producción de cal y cemento con su correspondiente distribución hacia los centros de comercialización, se empleó la base de datos Ecoinvent3 presente en el software SimaPro (Copyright Pré, 2019).

\section{TRABAJO DE CAMPO}

En aras de cuantificar el consumo de energía y recursos y los descartes residuales correspondientes a la etapa de fabricación de este tipo de bloques, se efectuaron visitas de campo a la empresa productora de BTC "Mobak" en las que se registraron in situ los datos requeridos.

En cada visita efectuada se tomó nota de la cantidad, proporción y origen de las materias primas usadas para producir los BTC, así como el destino final del producto ya manufacturado, con el fin de determinar las distancias de traslado. Se documentó el proceso de producción, especificando las etapas, máquinas y tipo de energía utilizada en cada una de fase del proceso. Se registró además el nombre, marca y modelo de la maquinaria empleada, su capacidad productiva y la eficiencia energética de cada operación expresada en unidades de energía. Asimismo, se registraron los distintos medios de transporte empleados, su capacidad de carga y las distancias recorridas.

En la Figura 1 se resume el proceso productivo aplicado por la empresa Mobak en la fabricación de sus BTC, donde, a diferencia de las dosificaciones propuestas para esta investigación, se incorporan dos tipos de arena con diferentes granulometrías y se añaden, tanto cal como cemento para la estabilización.

\section{RESULTADOS}

\section{RESISTENCIA A COMPRESIÓN}

En la Tabla 3 se detallan los resultados de los ensayos de resistencia a compresión realizados sobre cada una de las series de probetas: número de probetas ensayadas $(N)$, resistencia media a compresión $(\mu)$ y desviación estándar $(\sigma)$ de cada serie, el valor del estadístico $P$ resultante del análisis de varianzas y los factores de grupo del análisis de parejas de Tukey. En la Figura 2 se muestran las resistencias medias a compresión de cada serie de BTC producidos. 


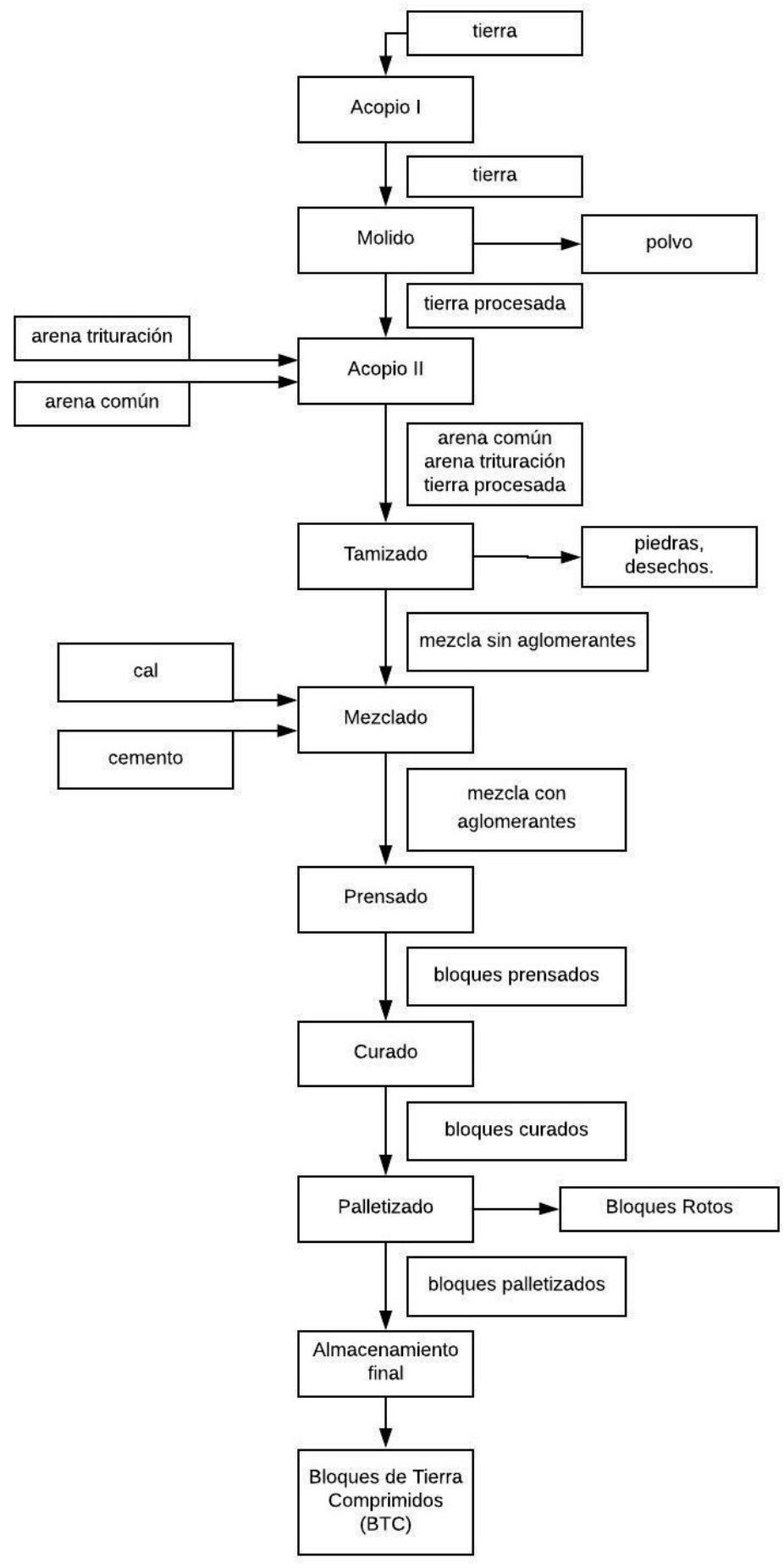

Figura 1. Diagrama de flujo de la producción de BTC. Fuente: Elaboración de los autores.

\section{TRABAJO DE CAMPO}

En la Tabla 4 se observa el consumo energético asociado a cada etapa del proceso productivo de los BTC. Estos consumos se obtuvieron tras relevar la planta de producción de la empresa Mobak: una producción media de 36 lotes por día, cada uno de ellos con 55 BTC. La asignación del consumo energético por unidad elemental (1 BTC) se realizó dividiendo el consumo eléctrico de todo el lote por 55 .

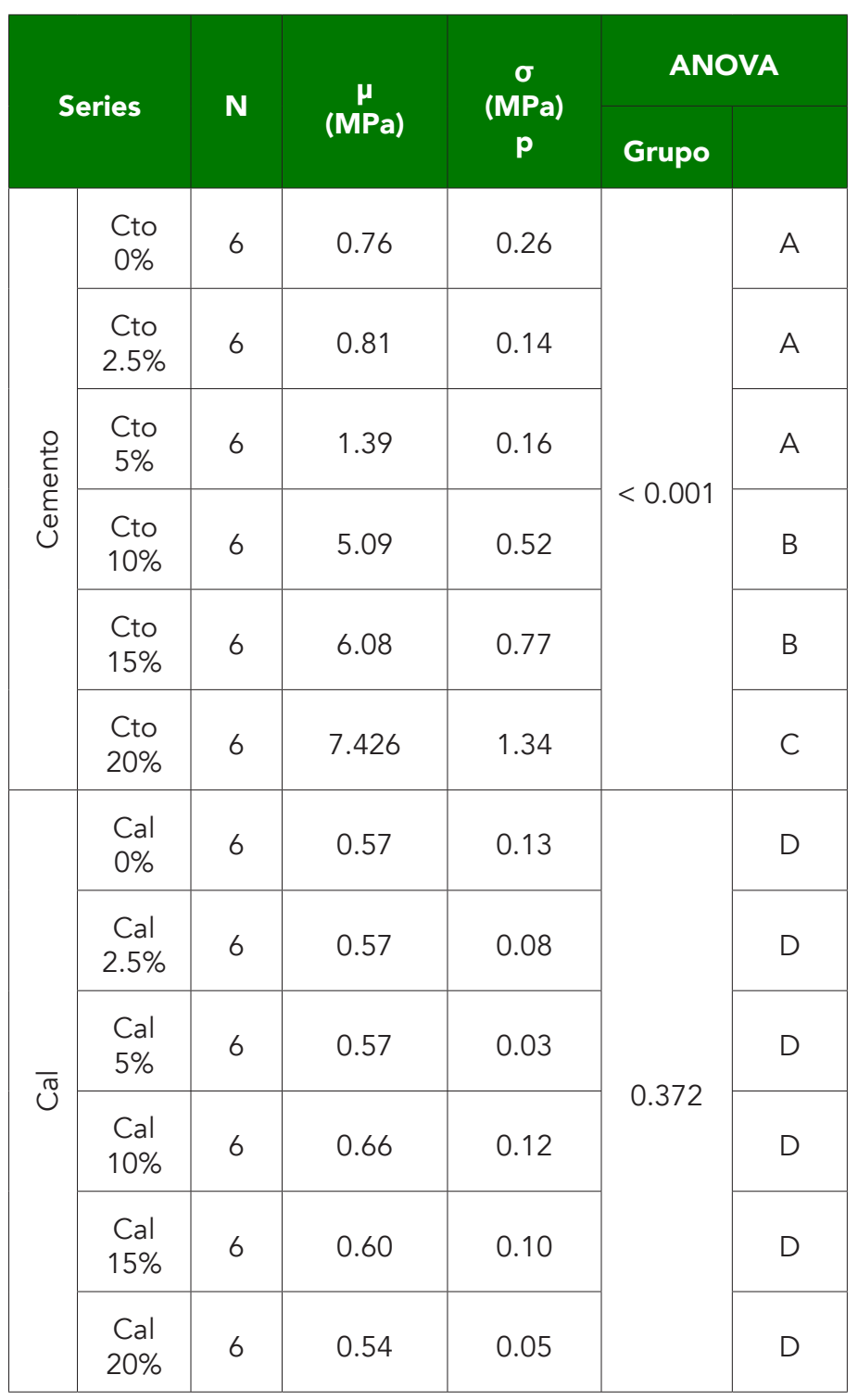

Tabla 3. Estadísticos descriptivos, análisis de varianza ANOVA y agrupamiento según análisis de Tukey de las series de BTC. Fuente: Elaboración de los autores.

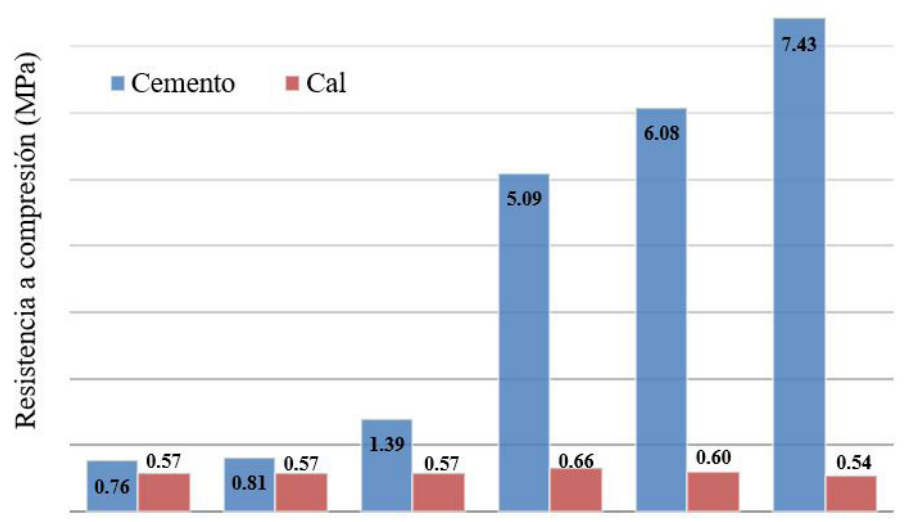

$\%$ Estabilizante

Figura 2. Resistencia a compresión de las diferentes series de BTC. Fuente: Elaboración de los autores. 


\begin{tabular}{|c|c|c|c|c|}
\hline $\begin{array}{c}\text { Consumo energético por } \\
\text { operación }\end{array}$ & $\begin{array}{c}\text { Potencia del } \\
\text { equipo }(\mathbf{K W})\end{array}$ & $\begin{array}{c}\text { Tiempo de uso por } \\
\text { lote (min) }\end{array}$ & $\begin{array}{c}\text { Energía consumida } \\
\text { por lote (MJ) }\end{array}$ & $\begin{array}{c}\text { Energía consumida } \\
\text { por BTC (KJ) }\end{array}$ \\
\hline Desterronadora & 2.0 & 8.5 & 1.020 & 0.0185 \\
\hline Tamizadora & 1.1 & 8.5 & 0.561 & 0.0102 \\
\hline Mezcladora & 5.5 & 7.0 & 2.310 & 0.0420 \\
\hline Cinta transportadora corta & 0.8 & 7.0 & 0.336 & 0.0061 \\
\hline Cinta transportadora larga & 1.1 & 7.0 & 0.462 & 0.0084 \\
\hline Prensa hidráulica & 3.0 & 8.5 & 1.530 & 0.0278 \\
\hline Curado & 0.4 & - & - & 0.0024 \\
\hline & & & TOTAL & 0.1155 \\
\hline
\end{tabular}

Tabla 4. Energía consumida en cada etapa del proceso de fabricación de 1 BTC. Fuente: Elaboración de los autores.

\begin{tabular}{|c|c|c|c|c|c|c|c|c|}
\hline Serie & $\begin{array}{l}\text { Acidificación } \\
\text { del suelo y } \\
\text { el agua }\end{array}$ & $\begin{array}{l}\text { Eutrofización } \\
\text { del agua }\end{array}$ & $\begin{array}{c}\text { Calentamiento } \\
\text { global }\end{array}$ & $\begin{array}{l}\text { Oxidación } \\
\text { fotoquímica }\end{array}$ & $\begin{array}{c}\text { Consumo } \\
\text { de } \\
\text { minerales }\end{array}$ & $\begin{array}{l}\text { Consumo de } \\
\text { combustibles } \\
\text { fósiles }\end{array}$ & $\begin{array}{l}\text { Consumo } \\
\text { de agua }\end{array}$ & $\begin{array}{l}\text { Deterioro } \\
\text { de la capa } \\
\text { de ozono }\end{array}$ \\
\hline & (kg SO2 eq) & (kg PO4 eq) & (kg CO2 eq) & $\begin{array}{c}\text { (kg } \\
\text { NMvOC) }\end{array}$ & $\begin{array}{c}\text { (kg Sb } \\
\text { eq) }\end{array}$ & (MJ) & (m3 eq) & $\begin{array}{l}\text { (kg CFC-11 } \\
\text { eq) }\end{array}$ \\
\hline Cal 0\% & 2,270E-04 & 4,733E-05 & 0,0419 & $2,427 \mathrm{E}-04$ & $1,110 \mathrm{E}-07$ & 0,6137 & 0,1085 & 5,761E-09 \\
\hline Cal 2,5\% & 3,530E-04 & 6,798E-05 & 0,1350 & 3,624E-04 & $1,242 \mathrm{E}-07$ & 1,0708 & 0,1104 & 1,130E-08 \\
\hline Cal 5\% & 4,790E-04 & 8,864E-05 & 0,2280 & 4,821E-04 & $1,374 \mathrm{E}-07$ & 1,5278 & 0,1123 & $1,685 \mathrm{E}-08$ \\
\hline Cal $10 \%$ & 7,311E-04 & 1,299E-04 & 0,4150 & 7,216E-04 & $1,639 \mathrm{E}-07$ & 2,4419 & 0,1161 & 2,793E-08 \\
\hline Cal 15\% & 9,831E-04 & 1,713E-04 & 0,6010 & $9,610 \mathrm{E}-04$ & $1,903 \mathrm{E}-07$ & 3,3559 & 0,1198 & 3,902E-08 \\
\hline Cal 20\% & 1,235E-03 & $2,126 \mathrm{E}-04$ & 0,7870 & $1,200 \mathrm{E}-03$ & $2,167 \mathrm{E}-07$ & 4,2700 & 0,1236 & $5,010 \mathrm{E}-08$ \\
\hline Cto $0 \%$ & 2,489E-04 & 5,420E-05 & 0,0453 & $2,650 \mathrm{E}-04$ & $1,296 \mathrm{E}-07$ & 0,6532 & 0,1579 & 6,063E-09 \\
\hline Cto $2,5 \%$ & 4,515E-04 & 1,019E-04 & 0,1380 & 4,443E-04 & $1,701 \mathrm{E}-07$ & 1,0228 & 0,1609 & 8,701E-09 \\
\hline Cto $5 \%$ & 6,541E-04 & 1,497E-04 & 0,2310 & 6,237E-04 & $2,105 \mathrm{E}-07$ & 1,3924 & 0,1639 & $1,134 \mathrm{E}-08$ \\
\hline Cto $10 \%$ & 1,059E-03 & $2,451 \mathrm{E}-04$ & 0,4160 & $9,825 \mathrm{E}-04$ & 2,913E-07 & 2,1316 & 0,1699 & 1,661E-08 \\
\hline Cto $15 \%$ & 1,038E-03 & $3,462 \mathrm{E}-04$ & 0,4920 & 1,017E-03 & 2,575E-07 & 1,6293 & 0,1569 & 1,361E-08 \\
\hline Cto $20 \%$ & 1,870E-03 & 4,360E-04 & 0,7870 & $1,700 \mathrm{E}-03$ & 4,530E-07 & 3,6100 & 0,1820 & 2,717E-08 \\
\hline
\end{tabular}




\section{IMPACTO AMBIENTAL}

A la hora de expresar los resultados del análisis de ciclo de vida realizado sobre las 12 series de BTC mediante el software Simapro, se utilizó el método de la EPD 2018 (Environmental Product Declarations) que cuantifica el impacto ambiental en 8 niveles de impacto, los cuales pueden apreciarse en la Tabla 5.

\section{DISCUSIÓN}

\section{SOBRE LA RESISTENCIA A COMPRESIÓN}

El efecto del contenido de estabilizante en la resistencia a compresión de las diferentes series de BTC presentó comportamientos diferentes según el tipo de aditivo empleado. Puede afirmarse, con un $95 \%$ de confianza, que la resistencia media a compresión de todas las series estabilizadas con cal, independientemente del porcentaje utilizado, es estadísticamente igual a la resistencia media a compresión de la serie de BTC sin estabilizar.

Por el contrario, la resistencia de los BTC con cemento se vio sumamente influenciada por el porcentaje de estabilizante empleado: las series de bloques estabilizadas con 2.5 y $5 \%$ de cemento presentaron, desde el punto de vista estadístico, una resistencia media a compresión sin diferencia significativa entre sí, e igual a la de los BTC sin estabilizante alguno (factor de grupo A). Los bloques con 10 y $15 \%$ de cemento presentaron resistencias estadísticamente iguales entre sí y superiores a las de sus homólogos con bajos contenidos de cemento (factor de grupo B), mientras que la serie estabilizada con $20 \%$ de cemento presentó una resistencia media a compresión superior a la de las demás series (factor de grupo C). La relación entre el contenido de cal y cemento empleados para la estabilización y la resistencia media a compresión de los BTC puede apreciarse en la Figura 3.

Los resultados obtenidos coinciden con los de investigaciones previas realizadas por el Laboratorio de Geotecnia de la Universidad Tecnológica Nacional, Facultad Regional Santa Fe (UTN FRSF), en las cuales se ha demostrado que la resistencia a compresión de los BTC estabilizados con cal es significativamente menor que la de sus homólogos estabilizados con igual proporción de cemento (Cabrera, González y Rotondaro, 2019), lo que, a su vez, coincide y refuerza los resultados generados por diferentes investigadores (González López, Juárez Alvarado, Ayub Francis y Mendoza Rangel, 2018; Laguna, 2011; Ouedraogo et al., 2020)

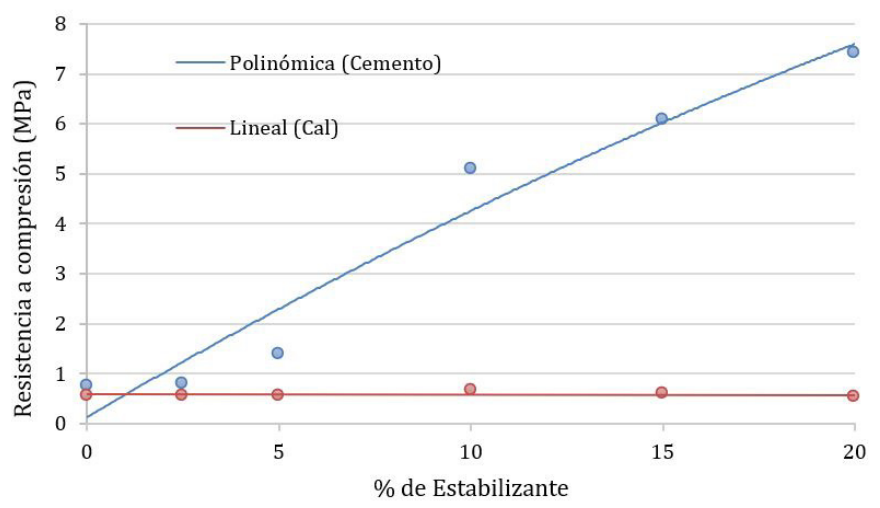

Figura 3. Curvas de interpolación resistencia a compresión vs. porcentaje de cal o cemento empleados en la estabilización de los BTC. Fuente: Elaboración de los autores.

\section{SOBRE EL IMPACTO AMBIENTAL}

En la Figura 4 se exponen de manera gráfica los resultados expresados en la Tabla 5 y obtenidos tras realizar el análisis de inventario de los BTC en estudio. En lo que respecta a la acidificación del suelo y el agua (1), la eutrofización del agua (2), la oxidación fotoquímica (4) y el consumo de minerales (5), el factor de impacto aumenta con el contenido de estabilizante, siendo en todos los casos mayor para los BTC estabilizados con cemento. Al contrario, el consumo de combustibles fósiles (6) y el deterioro de la capa de ozono (8) son significativamente mayores para los BTC estabilizados con cal que con cemento, lo cual no sucede con las emanaciones de $\mathrm{CO}_{2}$ equivalente (3), las cuales, a pesar de aumentar significativamente con el contenido del estabilizante empleado, se mantienen prácticamente igual para ambos estabilizantes.

Es interesante remarcar cómo el consumo de agua (7) es superior en los BTC estabilizados con cemento que en aquellos estabilizados con cal. Sin embargo, como puede apreciarse en el primer cuadrante de la Figura 4, esto no se debe al tipo de estabilizante empleado sino, al mayor contenido de arena usado en la fabricación de estos bloques, ya que su extracción demanda grandes volúmenes de agua en relación con los requeridos para explotación de una cantera de tierra.

La comparación entre energías incorporadas por diferentes investigadores no es tarea sencilla ya que, además de las diferentes materias primas y procesos de fabricación analizados, cada investigación posee objetivos, alcances e inventarios diferentes. En la Tabla 6, puede verse cotejada la energía incorporada 

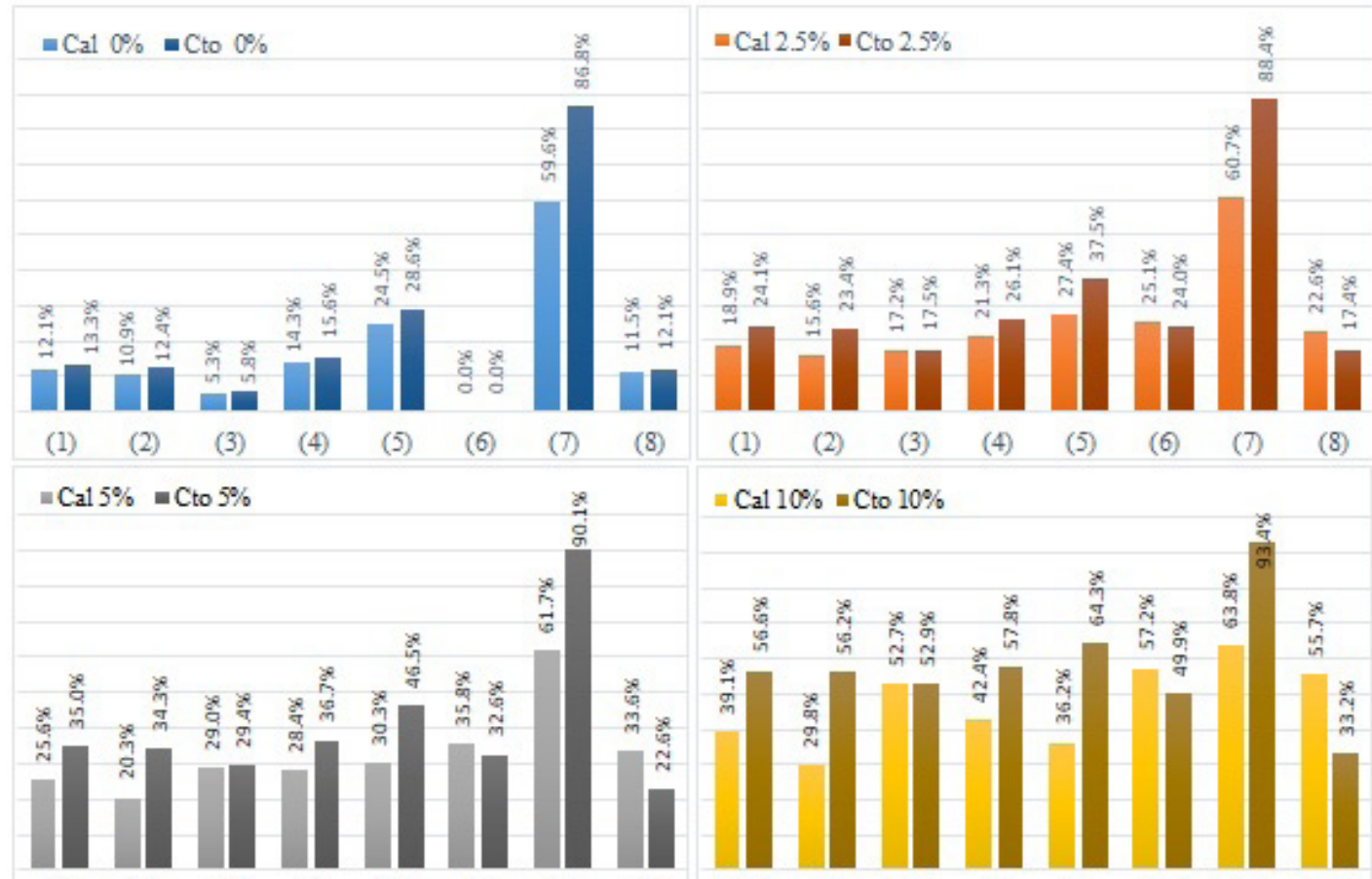

$\mathrm{Cal} 10 \%=\mathrm{C}$ to $10 \%$
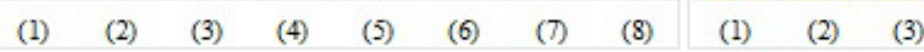

(3) (4)

4) (5)
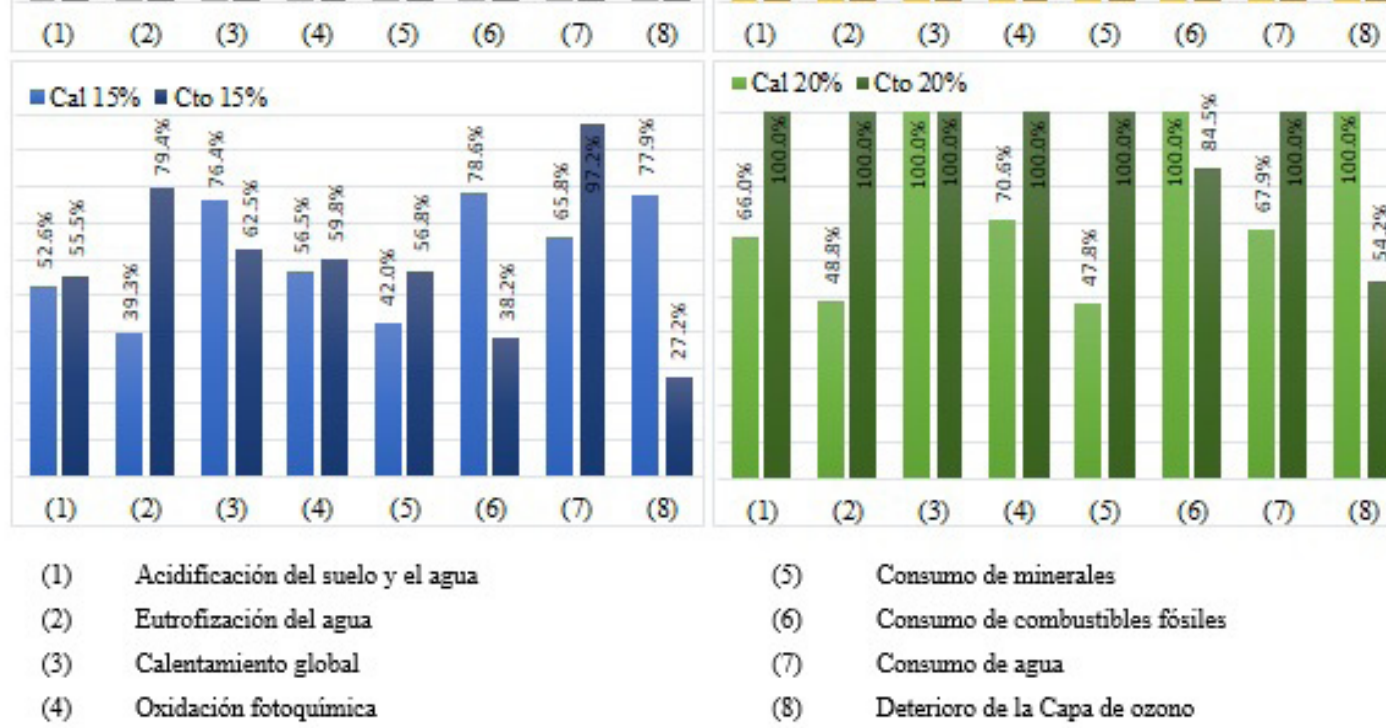

$=\mathrm{Cal} 20 \%$ - Cto $20 \%$
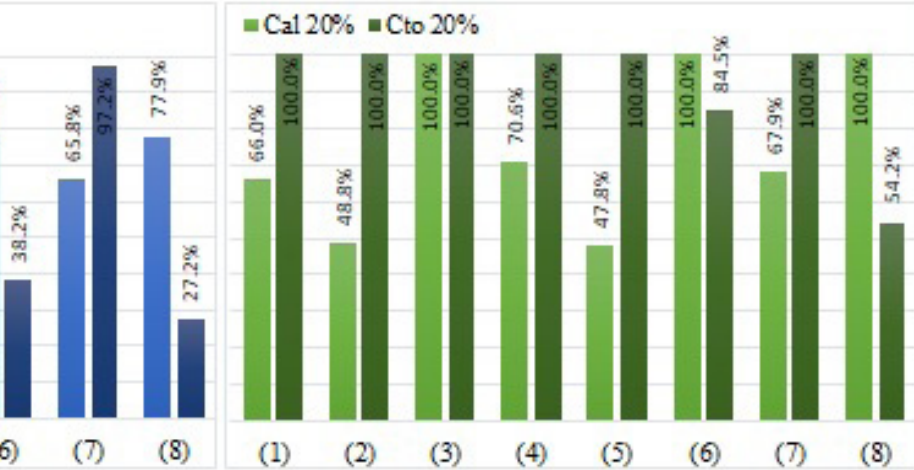

(8)

(1)

(2) (3)

(4)

(5)

(6) (7)

(8)

(1) Acidificación del suelo y el agua

(5) Consumo de minerales

(6) Consumo de combustibles fósiles

(4) Oxidación fotoquímica

(7) Consumo de agua

(8) Deterioro de la Capa de ozono

Figura 4. Comparación entre los diferentes factores de impacto de BTC estabilizados con cal y cemento, en diversas proporciones. Fuente: Elaboración de los autores.

\begin{tabular}{|c|c|c|}
\hline Autores & $\begin{array}{l}\text { Energía Incorporada } \\
\text { (MJ/BTC) }\end{array}$ & Estabilizante empelado \\
\hline Vázquez Espi, 2001 & $0.18-5.76$ & $\begin{array}{l}\text { Diferentes proporciones de } \\
\text { cemento Portland }\end{array}$ \\
\hline Roux Gutiérrez y Espuna Mujica, 2016 & 7.62 & 7\% de cal aérea hidratada \\
\hline $\begin{array}{l}\text { Fernandes, Peixoto, Mateus y Gervásio, 2019the } \\
\text { life cycle assessment of building materials is still in } \\
\text { its infancy. So far, there is only a small number of } \\
\text { Environmental Product Declarations (EPDs }\end{array}$ & 3.94 & $6.5 \%$ de cal hidráulica \\
\hline Presente investigación & $0.65-4,27$ & $\begin{array}{l}\text { Diferentes proporciones de cal } \\
\text { y cemento Portland }\end{array}$ \\
\hline
\end{tabular}


por las diferentes series de BTC analizadas en este trabajo -entre 0.65 y 4, 27 MJ/ BTC-, según la cantidad y tipo de estabilizante empleado-, y en función de los resultados publicados por diversos autores, cuya similitud queda allí manifiesta.

\section{CONCLUSIONES}

Tras evaluar los resultados de los ensayos de resistencia a compresión de las diferentes series de BTC fabricados en el Laboratorio de Geotecnia de la UTN FRSF y el impacto ambiental asociado a su producción, es posible establecer las siguientes conclusiones:

- La incorporación de cal aérea hidratada en la estabilización de los BTC no mejora su resistencia a compresión simple.

- La estabilización con cemento Portland aumenta significativamente la resistencia a compresión de los bloques, advirtiéndose una mejora considerable para porcentajes superiores al $5 \%$ en peso.

- La estabilización de los BTC con cal o cemento, aun en pequeñas cantidades, es la mayor responsable del impacto ambiental negativo de estos bloques.

- El reemplazo de cal por cemento en la estabilización de los BTC no disminuye de manera significativa el impacto ambiental vinculado a su producción.

Finalmente, puede afirmarse que, empleando suelos y arena característicos de la ciudad de Santa Fe (Argentina), estabilizados entre el $5 \%$ y el $10 \%$ en peso de cemento Portland, se alcanzan niveles de resistencia a compresión suficientes para cumplir funciones estructurales, y se minimiza el impacto ambiental asociado a este tipo de mampuestos. Se concluye, además, que la estabilización con cal no incrementa la resistencia a compresión de los BTC y acrecienta de manera significativa el impacto negativo de éstos sobre el medio ambiente.

En continuidad con esta investigación, se proyecta elaborar el Análisis de Ciclo de Vida de otros mampuestos de la región -como el bloque de hormigón o el ladrillo cerámico- comparando su impacto ambiental con el de los BTC estabilizados con 5 y 10\% de cemento, y su impacto económico en la construcción. Se podrá estudiar, asimismo, el efecto de la estabilización con cal aérea hidratada en la resistencia a compresión a largo plazo y la durabilidad de los BTC.

\section{AGRADECIMIENTOS}

Este trabajo se enmarca en el Proyecto de Investigación y Desarrollo denominado "Análisis de las propiedades del Bloque de Tierra Comprimida (BTC) estabilizado con cal" (MSUTIFE0004877TC), financiado por la Universidad Tecnológica Nacional (Argentina). Se agradece al Consejo Nacional de Ciencia y Técnica "CONACYT" (México) y al Consejo Nacional de Investigaciones Científicas y Tecnológicas "CONICET" (f) por financiar el trabajo de los autores, como también a la empresa "Mobak" por su colaboración.

\section{REFERENCIAS BIBLIOGRÁFICAS}

Angulo, D. E. y Carreño, K. (2017). El Bloque de Tierra Comprimido o BTC. Una alternativa de construcción para la arquitectura contemporánea. NODO, 12(23), 31-37. http:// revistas.uan.edu.co/index.php/nodo/article/view/655

Aranda Jiménez, Y. G. y Suárez-Domínguez, E. J. (2014). Efecto de la impermeabilidad del Mucílago de Nopal en bloques de tierra comprimidos. Nova Scientia, 6(11), 331-323.

Ben Mansour, M., Ogam, E., Jelidi, A., Cherif, A. S. y Ben Jabrallah, S. (2017). Influence of compaction pressure on the mechanical and acoustic properties of compacted earth blocks: An inverse multi-parameter acoustic problem. Applied Acoustics, 125, 128-135. DOI: https://doi.org/10.1016/j. apacoust.2017.04.017

Bradley, R. A., Gohnert, M. y Bulovic, I. (2018). Construction considerations for low-cost earth brick shells. Journal of Construction in Developing Countries, 23(1), 43-60. Recuperado de http://web.usm.my/jcdc/vol23_1_2018/ jcdc2018.23.1.3.pdf

Cabrera, S. y González, A. y Rotondaro, R. (2019). Bloques de tierra comprimida estabilizados con cal. Evaluación de dosificaciones y resistencia a la compresión. En Fundación Eco Urbano, III Encuentro Latinoamericano y Europeo de Edificaciones y Comunidades Sostenibles (EUROelecs 2019), 22 a 25 de mayo de 2019, Santa Fe, Argentina (pp. 141-148). DOI: https://doi.org/10.33414/ajea.3.633.2019

Cabrera, S., González, A. y Rotondaro, R. (2020). Resistencia a compresión en Bloques de Tierra Comprimida. Comparación entre diferentes métodos de ensayo. Informes de la Construcción, 72(560). DOI: https://doi.org/10.3989/ic.70462

Carretero-Ayuso, M. y García-Sanz-Calcedo, J. (2018). Comparison between building roof construction systems based on the LCA. Revista de la Construcción, 18(1), 123136. DOI: https://doi.org/10.7764/RDLC.17.1.123

Copyright Pré (2019). SimaPro 9. Recuperado de https:// simapro.com/ 
Curadelli, S., López, M., Piastrellini, R., Arena, P. y Civit, B. (2019). Estudio socioambiental de la producción de ladrillos artesanales en Mendoza desde la perspectiva del análisis de ciclo de vida. Mendoza: edUTecNe. Recuperado de http://190.114.221.84/handle/20.500.12272/3719

Elahi, T. E., Shahriar, A. R., Alam, M. K. y Abedin, M. Z. (2020). Effectiveness of saw dust ash and cement for fabrication of compressed stabilized earth blocks. Construction and Building Materials, 259. DOI: https://doi.org/https://doi. org/10.1016/j.conbuildmat.2020.120568

Fernandes, J., Peixoto, M., Mateus, R. y Gervásio, H. (2019). Life cycle analysis of Environmental impacts of earthen materials in the Portuguese context: Rammed earth and compressed earth blocks. Journal of Cleaner Production, 241. DOI: https://doi.org/10.1016/j.jclepro.2019.118286

González, A. y Cabrera, S. (2017). Prensa electromecánica para BTC. En Neves, C, Salcedo, Z. y Borges, O. (Eds.), XVII Seminario Iberoamericano de Arquitectura y Construcción con Tierra (SIACOT), (pp. 91-100). La Paz: PROTERRA. Recuperado de https://redproterra.org/wp-content/ uploads/2020/06/17-SIACOT-Bolivia-2017.pdf

González López, J., Juárez Alvarado, C., Ayub Francis, B. y Mendoza Rangel, J. (2018). Compaction effect on the compressive strength and durability of stabilized earth blocks. Construction and Building Materials, 163, 179-188. DOI: https://doi.org/10.1016/j.conbuildmat.2017.12.074

Guapi Cepeda, G. M. y Yagual Flores, K. D. (2017). Análisis de la producción de cal de la comuna San Antonio como oferta exportable a los mercados internacionales. Tesis de pregrado. Universidad de Guayaquil. Recuperado de http://repositorio.ug.edu.ec/handle/redug/47728

Guilarducci, A. (2018). Generación de adiciones minerales para el cemento Portland a partir de residuos de centrales termoeléctricas de lecho fluidizado. Tesis doctoral. Universidad Nacional del Litoral. Recuperado de https:// bibliotecavirtual.unl.edu.ar:8443/handle/11185/1103

Guzmán, S. e Iñiguez, M. (2016). Election methodology of chemical stabilizers for earth blocks. Estoa, 5(9), 151-159. DOI: https://doi.org/10.18537/est.v005.n009.12

Hegyi, A., Dico, C. y Catalan, G. (2016). Construction sustainability with adobe bricks type elements. Urbanism. Arhitectura. Constructii, 7(2), 147-156. Recuperado de https://pdfs.semanticscholar.org/4296/ f73ce17aad2539bda49d366ef7e2d08c93ed.pdf

Herrera Villa, J. (2018). Modelamiento numérico del comportamiento sísmico de viviendas de mampostería con bloques de tierra comprimida. Tesis de magíster. Pontificia Universidad Católica del Perú. Recuperado de http://tesis. pucp.edu.pe/repositorio/handle/20.500.12404/12059

IRAM (2017). IRAM-ISO 14040. Gestión ambiental. Análisis del ciclo de vida. Principios y marco de referencia. Buenos Aires.
Laborel-Préneron, A., Aubert, Jean-Emmanuel Magniont, C., Maillard, P. y Poirier, C. (2016). Effect of plant aggregates on mechanical properties of earth bricks. Journal of Materials in Civil Engineering, 29(12), 719-734. DOI: https://doi. org/10.1016/j.conbuildmat.2016.02.119

Laguna, M. (2011). Ladrillo Ecológico Como Material Sostenible para las Construcción. Trabajo final de Carrera. Universidad Pública de Navarra. Recuperado de http:// academica-e.unavarra.es/bitstream/handle/2454/4504/ 577656.pdf?sequence $=1$

Maddalena, R., Roberts, J. J. y Hamilton, A. (2018). Can Portland cement be replaced by low-carbon alternative materials? A study on the thermal properties and carbon emissions of innovative cements. Journal of Cleaner Production, 186, 933-942. DOI: https://doi.org/https://doi. org/10.1016/j.jclepro.2018.02.138

Malkanthi, S. N., Balthazaar, N. y Perera, A. A. D. A. J. (2020). Lime stabilization for compressed stabilized earth blocks with reduced clay and silt. Case Studies in Construction Materials, 12. DOI: https://doi.org/10.1016/j.cscm.2019. e00326

Neves, C. y Borges Farías, O. (Orgs.) (2011). Técnicas de Construcción con Tierra. Bauru: FEB-UNESP / PROTERRA. Recuperado: https://redproterra.org/wp-content/uploads/ 2020/05/4a_PP-Tecnicas-de-construccion-con-tierra_2011. pdf

ONNCCE (2015). NMX-C-508 Industria de la Construcción. Bloques de tierra comprimida estabilizados con cal. Especificaciones y métodos de ensayo. México D.F.

Ouedraogo, K. A. J., Aubert, J. E., Tribout, C. y Escadeillas, G. (2020). Is stabilization of earth bricks using low cement or lime contents relevant? Construction and Building Materials, 236. DOI: https://doi.org/10.1016/j. conbuildmat.2019.117578

Qiu, Q. (2020). A state-of-the-art review on the carbonation process in cementitious materials: Fundamentals and characterization techniques. Construction and Building Materials, 247. DOI: https://doi.org/https://doi. org/10.1016/j.conbuildmat.2020.118503

Roux Gutiérrez, R. y Espuna Mujica, J. (2016). El Hidróxido de Calcio y los bloques de tierra comprimida, alternativa sostenible de construcción. Nova Scientia, 5(2), 176-202. DOI: https://doi.org/10.21640/ns.v5i9.163

Sitton, J. D., Zeinali, Y., Heidarian, W. H. y Story, B. A. (2018). Effect of mix design on compressed earth block strength. Construction and Building Materials, 158, 124-131. DOI: https://doi.org/10.1016/j.conbuildmat.2017.10.005

Unión Europea (UE). (2007). Norma Euro 5. Bruselas.

Van Damme, H. y Houben, H. (2018). Earth concrete. Stabilization revisited. Cement and Concrete Research, 114, 90-102. DOI: https://doi.org/https://doi.org/10.1016/j. cemconres.2017.02.035 
Vázquez Espi, M. (2001). Construcción e impacto sobre el ambiente: el caso de la tierra y otros materiales. Informes de la Construcción, 52(471), 29-43. DOI: https://doi.org/10.3989/ic

Vissac, A., Bourges, A. y Gandreau, D. (2017). Argiles \& Biopolyméres. Les stabilisants naturaels pour la construction en terre. Grenoble: CRATerre éditions. Recuperado de https:// craterre.hypotheses.org/1370 